\title{
On the SuperDARN autocorrelation function observed in the ionospheric cusp
}

\author{
R. André, M. Pinnock, A.S. Rodger \\ Bristish Antarctic Survey, Natural Environment Research Council, Madingley Road, Cambridge CB3 OET, \\ UK
}

\begin{abstract}
The SuperDARN radars are proving to be a very powerful experimental tool for exploring solar windmagnetosphere-ionosphere interactions. They measure the autocorrelation function (ACF) of the transmitted signal backscattered from the ionospheric irregularities, and derive parameters such as the Doppler velocity and the spectral width. The associated spectra have a specific behaviour inside the cusp, a strong temporal and spatial evolution of the velocity and spectral width, and a high value of the spectral width. Although no studies have explained these characteristics, they are routinely used to detect the cusp in the radar data and estimate the open/closed field line boundary. Both satellite and magnetometer data show in the same region broadband wave activity in the Pc1, Pc2 frequency band. In this study, we evaluate how such wave activity modifies the radar's $\mathrm{ACF}$, and conclude that it explains the spectra seen in the cusp.
\end{abstract}

\section{Introduction}

Identification of the ionospheric signature of the cusp is important for many studies of solar wind-magnetosphereionosphere coupling processes. For example the cusp signature is a proxy for the open/closed field line boundary on the dayside, and flow across this boundary is a measure of the reconnection rate at the magnetopause [Baker et al., 1997]. There have been several proxies of the cusp such as the low altitude particle [Newell and Meng, 1988] and magnetometer [Menk et al., 1992] signatures. The HF radar signature has also been extensively used (eg Baker et al. [1997]) but the physical explanation has remained elusive; it is the topic of this paper.

From the transmission of a multiple pulse scheme, the SuperDARN radars [Greenwald et al., 1995] measure the autocorrelation (ACF) of the signal backscattered at several distances (range gates) from the radar by field-aligned electron concentration irregularities. In each range gate, this ACF is routinely analysed by a basic method (FITACF) [ Villain et al., 1987] which extracts the power, the line-of-sight Doppler velocity of the irregularities and the spectral width. Baker et al. [1995] have shown that the ACFs recorded in the cusp have a very particular behaviour. These studies used

Copyright 1999 by the American Geophysical Union.

Paper number 1999GL003658.

0094-8276/99/1999GL003658\$05.00
DMSP satellite data to identify the cusp from the particle characteristics [Newell and Meng, 1988]. They showed that the Doppler velocity spectrum observed in the cusp presents several overlapping components and that these spectra are not well-correlated from gate to gate (see Baker et al. [1995], Figure 2). The authors described these spectra as a "reflection of complex temporal and spatial behaviour of the structures involved in the scattering process".

Inside the cusp, the spectral width, as determined by FI$\mathrm{TACF}$, is very high (greater than $150 \mathrm{~ms}^{-1}$ ), and highly variable. When the spectrum contains several components, the FITACF method cannot resolve the width of the main component, but usually gives an over-estimation of it (i.e. the spectral width does not represent the full width at half power of a single component). The spectral width variability might then be assumed to be coming from the dynamics of the spectrum. For the same reason, the line-of-sight velocity is not constant, but varies around an average value. Published data show that the temporal velocity variation in one range gate is much higher inside the cusp than outside [Pinnock et al., 1995, Figure 2].

Although no studies have explained these behaviours, the cusp could easily be identified in the radar data by a high and variable spectral width, a variable velocity, and a large standard deviation associated to the linear fit performed on the phase.

Intense wave activity has been observed when satellites pass through the cusp (see for example Maynard et al. [1991]). Matsuoka et al. [1993], and later Erlandson and Anderson [1996], have clearly shown a step-like increase of wave activity in the frequency band $0.2-2.2 \mathrm{~Hz}$ with electric field values of few $\mathrm{mVm}^{-1}$, coincident with the cusp particle precipitation. These waves result from a superposition of electrostatic noise and electromagnetic waves.

Ground magnetometers do observe Pc1-2 waves near the cusp. But [Dyrud et al., 1997], noting the differences in occurence between satellite and ground data, suggested they are observing two separate phenomena.

In this paper, we evaluate the effect of such a time varying electric field (as observed by satellite) in the determination of the radar ACF, and demonstrate that the broadband wave activity in the Pc1-Pc2 frequency band can explain the ACF characteristics routinely observed in cusp.

\section{Time-varying electric field}

The satellite data clearly show the onset of strong wave activity in the cusp, in the frequency band $0.1-5 \mathrm{~Hz}$, and that 
the wave amplitude can easily reach a few $\mathrm{mVm}^{-1}$. This implies an amplitude of the velocity field fluctuation greater than $50 \mathrm{~ms}^{-1}$. This contrasts with the background convection velocity which may give rise to line-of-sight velocity components of $1000 \mathrm{~ms}^{-1}$. Here we study in more detail the effect of the wave activity on the radar ACFs.

To compute the ACF the SuperDARN radar emits a pattern of pulses which are not evenly spaced. It receives the backscattered signal from ionospheric irregularities [Greenwald et al., 1985]. After about $75 \mathrm{~ms}$, the radar halts sounding and computes the ACF of the signal backscattered from each range. Then, in order to reduce the noise level, the radar integrates this ACF over 70 identical cycles (for the normal $7 \mathrm{~s}$ integration time). One can consider that each individual ACF (before the integration) contains the line-ofsight velocity information. If we assume that the velocity field varies between these cycles, then the integrated ACF will reflect the velocity distribution found during the integration time. For example, if we consider the presence of a monochromatic wave with a frequency of the order of 0.5 $\mathrm{Hz}$, one finds that the Fourier transform of the resulting ACF should exhibit two main components. This mechanism suggests that such a time-varying electric field could affect the spectrum, and gives rise to the observed multi-component spectra in the cusp. In essence, the problem is one of undersampling (the radar is sampling at less than the Nyquist frequency). The combination of the low amplitude of the wave compared with the line-of-sight velocity, the averaging of the ACFs over the integration period, the radar halt period for signal processing (which introduces a variable time delay in the sampling) and the precise details of the software algorithm that process the ACFs to derive the velocity and spectral widths (FITACF) make it difficult to predict exactly what the impact of such wave activity is.

To address this question, we have built a simulator. We consider only one range gate, in which all the scatterers are randomly distributed. All the scatterers are totally correlated, and there is no turbulence effect in this simulation. Thus for a constant velocity field, we have zero spectral width. For each pulse, the irregularities will backscatter the signal $\mathrm{S}(\mathrm{t})$ given by (1):

$$
\begin{gathered}
S(t)=\sum_{i} e^{-j \cdot \vec{k} \cdot \vec{r}_{i}(t)} \\
\vec{r}_{i}(t)=\vec{r}_{0}+\vec{V}(t) . t \\
\vec{V}(t)=\vec{V}_{0}+\vec{V}_{1} \cdot \sum_{k=1}^{500} \cos \left(2 . \pi \cdot f_{k} \cdot t+\phi_{k}\right)
\end{gathered}
$$

where $\vec{k}$ is the wave vector, $\vec{r}_{i}(\mathrm{t})$ is the position of the irregularity, $\vec{r}_{0}$ is the position at $\mathrm{t}=0$ and $\vec{V}(t)$ is the velocity coming from both the large-scale convection $\left(\vec{V}_{0}\right)$ and the wave experienced in the cusp. This wave is defined by a superposition of 500 monochromatic sources which have (i) a Gaussian frequency distribution, centred around a mean value and with a defined bandwidth, (ii) a uniform distribution of phases and (iii) a common amplitude $\left(\vec{V}_{1}\right)$. This large number of sources should give us a representative signature of the wave activity seen inside the cusp.
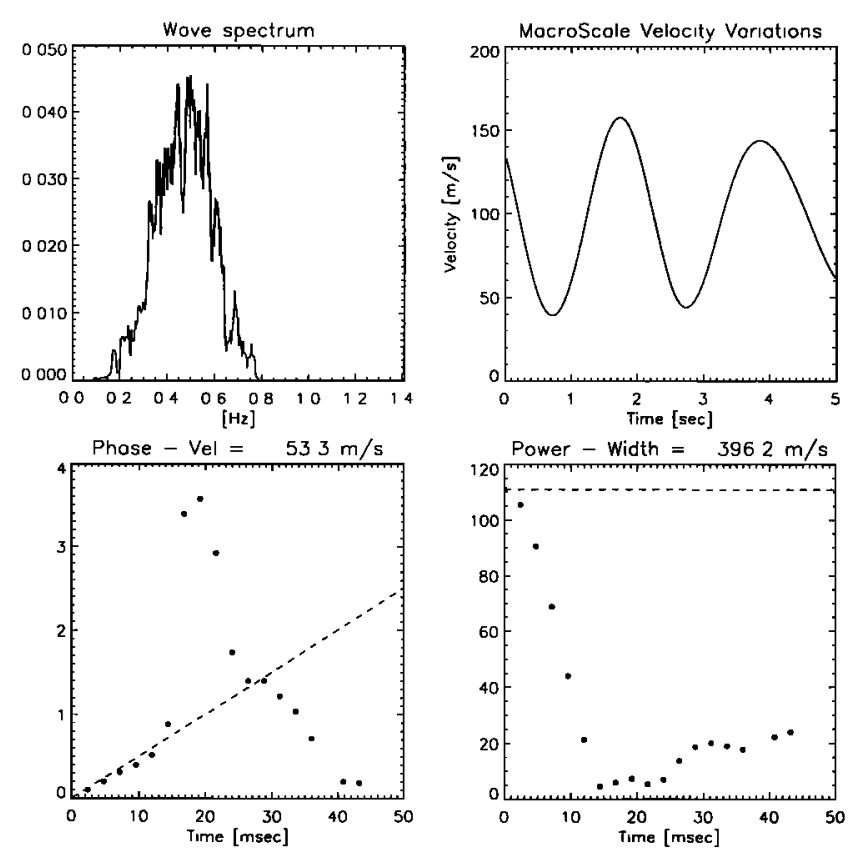

Figure 1. upper left-hand panel: spectrum of the wave used, upper right-hand panel: velocity variation during the radar integration time, lower panels: phase (left-hand) and power (right-hand) of the simulated ACF.

From this, we are able to compute the individual ACFs, to average them over the integration period, and then submit the final ACF to the routine fitting procedure (FITACF) to determine velocity and spectral width in an identical way to the SuperDARN observations.

\section{Simulation results}

The results presented here are characteristic of the radar running in a high-resolution mode, with an integration time of $5 \mathrm{~s}$ only, but are found to be identical for a radar running in the normal mode ( $7 \mathrm{~s}$ integration time).

Figure 1 gives an example of a simulated ACF when considering a broadband wave which has an amplitude, a mean frequency and a bandwidth of $40 \mathrm{~ms}^{-1}$ (which corresponds to an electric field of $2 \mathrm{mVm}^{-1}$ ), $0.5 \mathrm{~Hz}$, and $0.1 \mathrm{~Hz}$ respectively, and a background line-of-sight velocity of 100 $\mathrm{ms}^{-1}$. The first panel (upper left-hand corner) shows the wave frequency spectrum. The induced velocity variation during the integration time is plotted in the upper right-hand panel. The phase and the power of the ACFs are plotted on the lower left-hand and right-hand panels respectively, where the dotted lines present the results obtained without the velocity variations. One can see that the temporal evolution of the phase is not linear (as it would be for a single velocity component, c.f. Villain et al. [1987]), and that it clearly shows the presence of several components in the associated spectrum. This modulation is also reproduced on the temporal evolution of the power, as expected in such a case. The rapid decorrelation of this function results in a spectral width which has a value of about $300 \mathrm{~ms}^{-1}$, as de- 


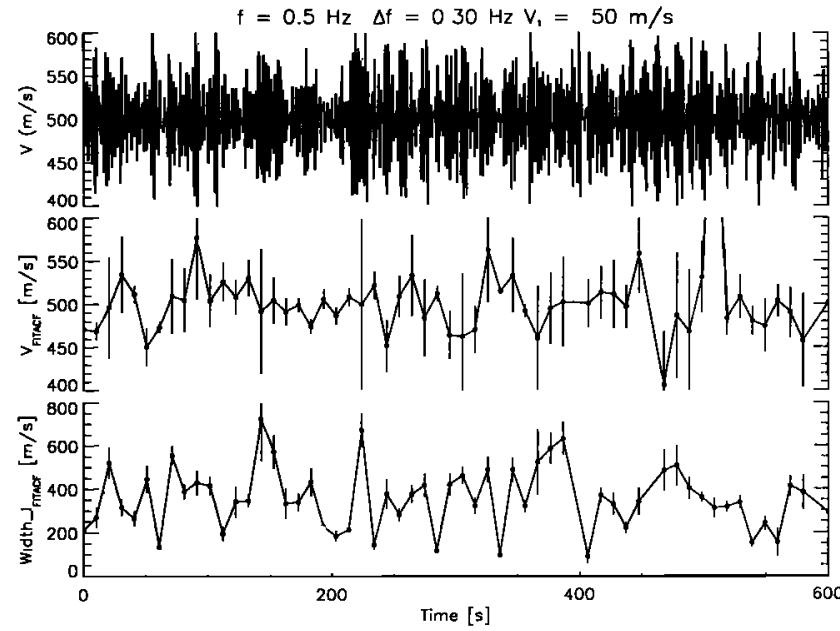

Figure 2. From top to bottom, time series of the wave induced velocity field, simulated line-of-sight velocity field and spectral width determined from the ACFs

termined by FITACF. This shows that such a wave is able to reproduce both the multi-component spectra and a high spectral width value, but still maintain the mean Doppler velocity.

With this wave model, we can produce a long time series of the velocity variations (here 10 minutes), then compute the ACFs recorded by the radar (running in the highresolution mode: $5 \mathrm{~s}$ integration time, with a temporal resolution of $10 \mathrm{~s}$ ), and apply the FITACF analysis to them. Figure 2 shows on the upper panel an example of timevarying electric field generated by this simulation. Here, the mean wave amplitude, wave frequency and bandwidth are $2.5 \mathrm{mVm}^{-1}$ (which corresponds to a velocity of $50 \mathrm{~ms}^{-1}$ ), $0.5 \mathrm{~Hz}$ and $0.3 \mathrm{~Hz}$ respectively. The two other panels show the Doppler velocity (middle panel) and the spectral width (bottom panel). The error bars show the statistical uncertainties associated to these parameters. This figure illustrates well the variability obtained in both the Doppler velocity and the spectral width.

Figure 3 shows the line-of-sight velocity (upper panel) and the spectral width (lower panel) recorded at Halley dur-

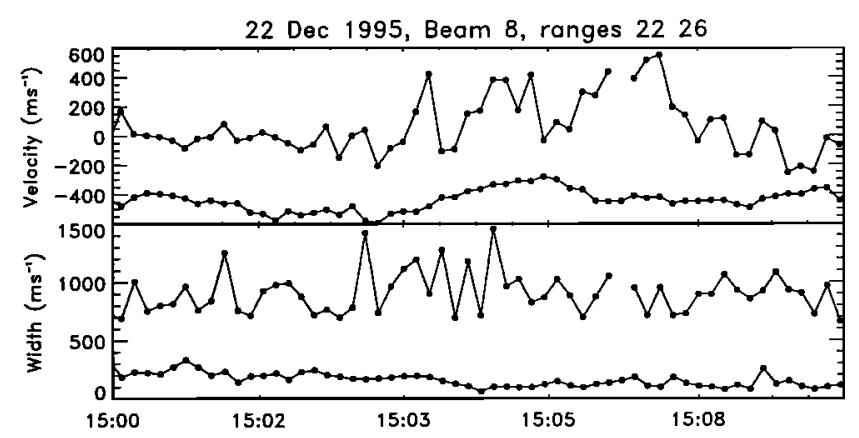

Figure 3. Velocity (upper panel) and spectral width (lower panel) recorded at Halley on the 22 December 1995, inside the cusp (upper curve) and equatorward of it (lower curve). The upper curves have been shifted by $500 \mathrm{~ms}^{-1}$ for clarity.

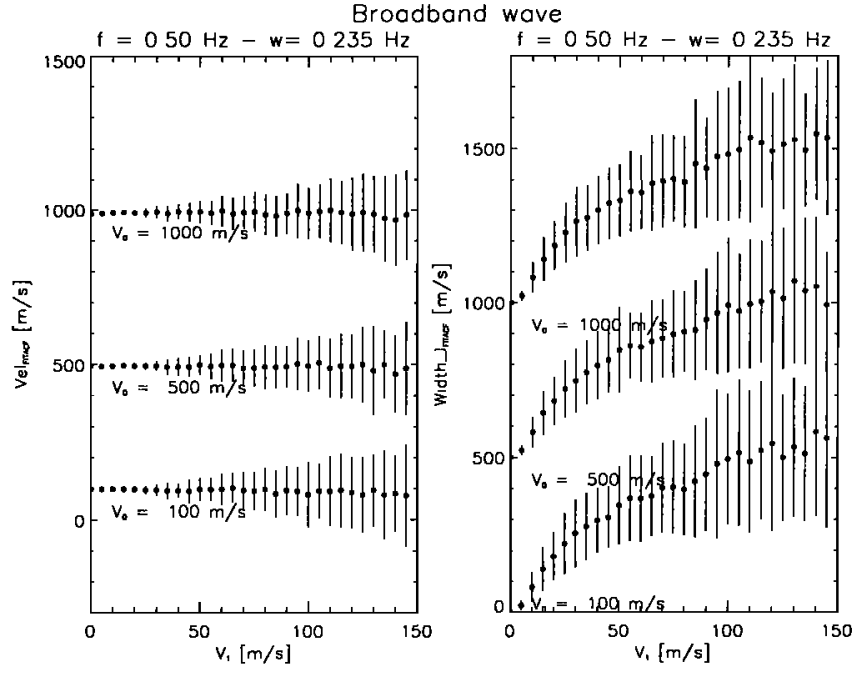

Figure 4. Mean value of the velocity (left) and the spectral width (right) as a function of the wave amplitude and for several background velocity. The error bars gives the standard deviations associated with the mean value.

ing 10 minutes, in two different range gates located inside and outside the cusp for the upper and lower curve respectively. The upper curve has been shifted by $500 \mathrm{~ms}^{-1}$ for clarity. This typical example shows a very good agreement between the simulated (figure 2) and recorded data.

From such a time series, one can compute the mean Doppler velocity and spectral width with their corresponding standard deviations. Figure 4 shows in the left panel the mean velocity as a function of the wave amplitude (frequency: 0.5 $\mathrm{Hz}$, bandwidth: $0.235 \mathrm{~Hz}$ ), for several values of the background line-of-sight velocity. The error bars represent the standard deviation of these averaged values, and so give us an estimate of the velocity fluctuations. The conclusion reached is that the mean velocity corresponds to the background velocity whatever the wave amplitude, but that the velocity fluctuations increase with the wave amplitude.

The right panel shows the spectral width (determined by applying a Lorentzian fit on the ACF power: width_l) as a function of the wave amplitude and for several background velocities. One has to note that the curves which correspond to a background velocity of $500 \mathrm{~ms}^{-1}$ and $1000 \mathrm{~ms}^{-1}$ have been shifted by $500 \mathrm{~ms}^{-1}$ and $1000 \mathrm{~ms}^{-1}$ respectively for clarity. The figure shows that, regardless of the background line-of-sight velocity value, a small wave amplitude (1.25 $\mathrm{mVm}^{-1}=25 \mathrm{~ms}^{-1}$ ) can result in a spectral width value of $200 \mathrm{~ms}^{-1}$ or greater. Again, the fluctuations of the determined spectral width increase with the wave amplitude.

\section{Conclusion}

In this study, we have examined the impact of the wave activity, routinely observed in the cusp, with electric field variations in the 0.1-5 $\mathrm{Hz}$ frequency band, and with amplitudes of few $\mathrm{mV} \cdot \mathrm{m}^{-1}$. To do this we have simulated the effect of such a time varying electric field on the radar $\mathrm{ACF}$, and then 
in the parameters (line-of-sight velocity and spectral width) deduced from the standard analysis method (FITACF).

We have shown that this wave activity induces both a large spectral width, a great variability in the velocity and in the spectral width, and a multi-component spectrum. These results are valid even for a small wave amplitude $\left(1 \mathrm{mVm}^{-1}\right)$, and are enhanced with the wave amplitude. One concludes that all the characteristics of the spectra recorded in the cusp can be explained by the presence of a broadband wave in the $\mathrm{Pc} 1-\mathrm{Pc} 2$ frequency band.

As a result, the large spectral width values that have been used by many authors as a very important diagnostic of the cusp in SuperDARN radar data can now be placed on a firm physical basis for the first time. The technique will be extended to compare carefully the cusp localization as determined by magnetometers and HF-radars, and to investigate the large spectral width observed on the nightside [Dudeney et al., 1998].

Acknowledgments. The authors acknowledge helpful discussions with Dr J.-P. Villain and Dr C. Hanuise. This work has been funded by the European Community grant ERB4001GT973635.

\section{References}

Baker, K.B., J.R. Dudeney, R.A. Greenwald, M. Pinnock, P.T. Newell, A.S. Rodger, N. Mattin, and C.-I. Meng, HF radar signatures of the cusp and low-latitude boundary layer, J. Geophys. Res., 100, 7671-7695, 1995.

Baker, K.B., A.S. Rodger, and G. Lu, HF-radar observations of the dayside magnetic merging rate: A geospace environment modeling boundary layer campaign study, J. Geophys. Res., 102, 9603-9617, 1997.

Dudeney, J.R., A.S. Rodger, M.P. Freeman, J. Pickett, J. Scudder, G. Sofko, and M. Lester, The nightside ionospheric response to IMF By changes, Geophys. Res. Lett., 25, 2601-2604, 1998.

Dyrud, L.P., M.J. Engebretson, J.L. Posh, W.J. Hugues, H. Fukunishi, R.L. Arnoldy, P.T. Newell, and R.B. Horne, Ground observations and possible source regions of two types of $\mathrm{pcl-2}$ micropulsation at very high-latitudes, J. Geophys. Res., 102, 27,011-27,027, 1997.

Erlandson, R.E., and B.J. Anderson, Pcl waves in the ionosphere: A statitical study, J. Geophys. Res., 101, 7843-7857, 1996.

Greenwald, R.A., K.B. Baker, R.A. Hutchins, and C. Hanuise, An HF phased array radar for studying small-scale structure in the high-latitude ionosphere, Radio Sci., 20, 63-79, 1985.

Greenwald, R.A., W.A. Bristow, G.J. Sofko, C. Senior, J.-C. Cerisier, and A. Szabo, Super dual auroral radar network radar imaging of dayside high latitude convection under northward interplanetary magnetic field: Toward resolving the distorted twocell versus multicell controversy, J. Geophys. Res., 100, 19,66119,674, 1995.

Matsuoka, A., K. Tsuruda, H. Hayakawa, T. Mukai, A. Nishida, T. Okada, N. Kaya, and H. Fukunishi, Electric field fluctuations and charged particle precipitation in the cusp, J. Geophys. Res., 98, 11,225-11,234, 1993.

Maynard, N.C., T.L. Aggson, E.M. Basinka, W.J. Burke, P. Craven, W.K. Peterson, M. Sugiura, and D.R. Weimer, Magnetospheric boundary dynamics: DE-1 and DE-2 observations near the magnetopause and cusp, J. Geophys. Res., 96, 3505-3522, 1991.

Menk, F.W., B.J. Fraser, H.J. Hansen, P.T. Newell, C.-I. Meng, and R.J. Morris, Identification of the magnetospheric cusp and cleft using pc1-2 ulf pulsations, J. Atmos. Terr. Phys., 54, 1021-1042, 1992.

Newell, P.T., and C.-I. Meng, The cusp and the cleft boundary layer: Low altitude identification and statistical local time variation, J. Geophys. Res., 93, 14,549-14,556, 1988.

Pinnock, M., A.S. Rodger, J.R. Dudeney, F. Rich, and K. Baker, High-spatial and temporal observations of the ionospheric cusp, Ann. Geophys., 13, 919-925, 1995.

Villain, J.-P., R.A. Greenwald, K.B. Baker, and J.M. Ruohoniemi, $\mathrm{HF}$ radar observations of $\mathrm{E}$ region plasma irregularities produced by oblique electron streaming, J. Geophys. Res., 92, 12,32712,342, 1987.

R. André, M. Pinnock and A.S. Rodger, British Antarctic Survey, High Cross, Madingley Road, CB3 OET Cambridge, United Kingdom

(Received August 17, 1999; accepted September 21, 1999.) 